\title{
Ecological Assembly Processes of the Bacterial and Fungal Microbiota of Wild and Domesticated Wheat Species
}

\author{
M. Amine Hassani, ${ }^{1,2, \dagger}$ Ezgi Özkurt, ${ }^{1,2}$ Sören Franzenburg, ${ }^{3}$ and Eva H. Stukenbrock ${ }^{1,2, \dagger}$ \\ ${ }^{1}$ Environmental Genomics, Christian-Albrechts University of Kiel, Am Botanischen Garten 1-9, 24118 Kiel, Germany \\ 2 Environmental Genomics, Max Planck Institute for Evolutionary Biology, August-Thienemann 2, 24306 Plön, Germany \\ ${ }^{3}$ Institute of Clinical Molecular Biology, Christian-Albrechts University of Kiel, Am Botanischen Garten 11, 24118 Kiel, Germany
}

Accepted for publication 31 March 2020.

\section{ABSTRACT}

Domestication has led to substantial changes in plant physiology. How this anthropogenic intervention has contributed in altering the wheat microbiota is not well understood. Here, we investigated the role of ecological selection, drift, and dispersal in shaping the bacterial and fungal communities associated with domesticated wheat Triticum aestivum and two wild relatives, $T$. boeoticum and $T$. urartu. Our study shows that the bacterial and fungal microbiota of wild and domesticated wheat species follow distinct community assembly patterns. Further, we revealed a more prominent role of neutral processes in the assembly of the microbiota of domesticated wheat and propose that domestication has relaxed selective processes in the assembly of the wheat microbiota.

Keywords: microbiome, neutral processes, niche processes, rhizosphere and phyllosphere, wheat domestication, wheat microbiota, wheat mycobiota
Early crop cultivation and domestication were key in the rise of agrarian societies and civilizations (Diamond 2002). Wheat represents one of the first cereal crop species, domesticated 10,000 years ago in the Fertile Crescent, it underwent through a reticulated evolution (Pont et al. 2019). The (un)conscious anthropogenic selection led to substantial alterations of the plant physiology that are hallmarked by seed size enlargement (Purugganan and Fuller 2009), loss of shattering (Fuller 2007), and increased yield (Preece et al. 2017). Archaeobotanical studies have evidenced that the spread of cereal crops from their center of origin through human migration and trade further contributed to their diversification

${ }^{\dagger}$ Corresponding authors: E. H. Stukenbrock; estukenbrock@bot.uni-kiel.de, and M. A. Hassani; hassani.medamine@gmail.com

Funding: The project was funded by the DFG Collaborative Research Centerre (CRC) 1182 "Origin and Function of Metaorganisms" and the Canadian Institute for Advanced Research, CIFAR

Author contributions: E.H.S. and M.A.H. designed the experiments. M.A.H. conducted the experiments, collected the results, and analyzed the data. E.Z. collected T. aestivum seeds from the province of Kislak Antakya in Turkey. S.F. performed the sequencing. M.A.H. and E.H.S. wrote the manuscript.

*The $e$-Xtra logo stands for "electronic extra" and indicates that supplementary materials are published online.

The author(s) declare no conflict of interest.
(Purugganan and Fuller 2009). Concomitantly, these multistep and sequential processes caused genetic bottlenecks and reduced genetic diversity in the genome of domesticated crops (Doebley et al. 2006).

Investigating domesticated plant species has been key to comprehend evolutionary processes that contributed to plant trait emergence (Purugganan and Fuller 2009) and their molecular genetic basis (Doebley et al. 2006). However, our understanding of the impact of domestication on the plant associated microbiota remains incipient. By comparing wild and modern crop genotypes, few studies have addressed the effect of domestication in altering the composition and structure of the plant-associated bacterial and fungal microbiota, (recently reviewed in Cordovez et al. 2019; Escudero-Martinez and Bulgarelli 2019; Hassani et al. 2019). Depending on the crop species, plant domestication has resulted in microbial diversity reduction (Zachow et al. 2014), reduced symbiotic interactions (Kiers et al. 2007), or depletion of a microbial phylogroup (Pérez-Jaramillo et al. 2018). While genetic diversity of crop species is reduced at a population level, we still lack insight why individual crop plants assemble less diverse microbial communities compared with their wild relatives. Furthermore, it remains unclear to what extent plant domestication has altered ecological processes (i.e., selection, drift, and dispersal) of the microbiota assembly. Based on detailed analyses of microbial communities, we propose here that wild and domesticed wheat species show distinct assembly patterns of bacterial and fungal microbiota and that selection processes are less eminent in the microbiota assembly of the domesticated wheat Triticum aestivum. To characterize the role of 
ecological drift, dispersal, and selection in governing the assembly of the wheat microbiota, we studied the bacterial and fungal microbiota of the domesticated wheat species T. aestivum and two of its wild relatives (i.e., T. boeoticum and T. urartu). In our analyses and interpretations, we followed the conceptual framework of microbial community assembly that pose ecological drift, selection, and dispersal as processes acting in concert, and not in disjoint, to drive microbial patterns (Herrera Paredes and Lebeis 2016; Nemergut et al. 2013; Vellend et al. 2014).

\section{MATERIALS AND METHODS}

Soil sample and wheat accessions. The soil was collected during summer 2017 from the experimental farm Hohenschulen (field Scheunenkopp, $\left.54^{\circ} 18^{\prime} 53.7^{\prime \prime} \mathrm{N}, 9^{\circ} 58^{\prime} 44.1^{\prime \prime} \mathrm{E}\right)$ of the ChristianAlberchts University of Kiel, Germany. After clearing off the surface, soil was taken from the top layer $(\sim 20 \mathrm{~cm})$. The soil was homogenized, passed through $5 \mathrm{~mm}$ sieve, spiked with $5 \%$ (vol/vol) of peat (HAWITA GmbH, Germany), and stored at room temperature for three additional months. The domesticated wheat, T. aestivum, used in this study, is a landrace that originates from the province of Kislak Antakya in Turkey $\left(35^{\circ} 58^{\prime} 21.8^{\prime \prime} \mathrm{N}\right.$, $36^{\circ} 07^{\prime} 41.8^{\prime \prime} \mathrm{E}$ ). Wild wheat varieties (i.e., T. boeoticum TRI 18344 and $T$. urartu TRI 18414) originate from Turkey and were kindly provided by the IPK Gatersleben, Germany.

Growth conditions. Prior to sowing the seeds, the seeds were surface sterilized through three successive washing steps. Seeds were washed as follows: (i) $3 \times$ with double distilled (dd) $\mathrm{H}_{2} \mathrm{O}$ (Veolia water technologies), (ii) $1 \times$ with sterile $1 \%$ phosphatebuffered saline ([PBS] vol/vol) supplemented with $0.02 \%$ (vol/vol) Tween 20 , (iii) $3 \times \mathrm{ddH}_{2} \mathrm{O}$, (iv) $1 \times$ with $80 \%$ (vol/vol) ethanol, and rinsed (v) $3 \times$ with $\mathrm{ddH}_{2} \mathrm{O}$. All steps were carried out using sterile 50-ml sterile tubes (Sarstedt AG \& Co., Germany). Each individual seed was sowed in square PVC pots containing $415 \mathrm{~g}$ of Kiel agricultural soil. All pots containing the same wheat genotype were placed in the same tray and incubated for an additional 10 days in a phytochamber with $16 / 8 \mathrm{~h}$ light $\left(\sim 200 \mu \mathrm{mol} / \mathrm{m}^{2} / \mathrm{s}\right) /$ dark cycle, $20^{\circ} \mathrm{C}$, and $90 \%$ relative humidity as growth conditions. Plants were watered with sterile tap water every other day by tray flooding. Unplanted soil pots were randomly distributed across the three trays.

Leaf, root, and rhizosphere sampling. Leaf, root, and rhizosphere samples were harvested for each individual plant separately and kept so for all processes from DNA extraction to PCR amplicon sequencing. The first leaf $(7 \mathrm{~cm})$ for each plant was cut and subjected to successive washing using $20 \mathrm{ml}$ of (i) $\mathrm{ddH}_{2} \mathrm{O}$, (ii) $1 \%$ phosphate-buffered saline (PBS, vol/vol) supplemented with $0.02 \%$ (vol/vol) Tween 20, and (iii) 1\% PBS. Leaves were briefly dried using sterile blotting paper ( $0.35 \mathrm{~mm}$, Hahnemühle, Germany). The remaining plant was gently dug out from soil-containing pot and shaken by hand to remove loosely attached soil. Below-ground tissue was separated from the above part, placed in 50-ml sterile tube (Sarstedt AG \& Co, Germany), and $20 \mathrm{ml}$ of $1 \%$ PBS was added to it. To recover the rhizosphere, the tube was mixed by vortex for $30 \mathrm{~s}$ at max speed. Recovered rhizosphere soil was resuspended in $3 \mathrm{ml}$ of sterile $\mathrm{dd}_{2} \mathrm{O}$. Corresponding roots were additionally washed following same the procedures as indicated above. Each leaf, root, and rhizosphere sample was transferred to a separate Lysing Matrix E tubes (MP Biomedicals, Santa Ana, CA, U.S.A.), snap frozen in liquid nitrogen, and then stored at $-80^{\circ} \mathrm{C}$. To maximize recovery of DNA yield, each sample was separated into three subsamples prior to their transfer to lysing tubes.

DNA extraction. Recovered samples were homogenized twice by Precellys 24 TissueLyser [6,300 rpm/15 s/10 s pause] (Bertin Technologies, Germany). The DNA extractions were conducted according to the manufacturer's protocol (FastDNA SPIN kit for Soil; MP Biomedicals), except for two additional steps that were included after homogenization. Samples were pretreated with (i) $36 \mu l$ of Lysozyme ( $1 \%$, wt/vol, in $1 \%$ PBS at $37^{\circ} \mathrm{C}$ for $30 \mathrm{~min}$ with intermittent shaking) (Merck KGaA, Germany) and then with (ii) $15 \mu \mathrm{l}$ of Proteinase K (>600 mA/ml) and $5 \mu \mathrm{l}$ of Rnase A (100 mg/ $\mathrm{ml}$ ) (Qiagen, Germany) for $5 \mathrm{~min}$ at room temperature. DNA was eluted in nuclease-free water (Merck KGaA, Germany) and stored at $-20^{\circ} \mathrm{C}$ for downstream processing.

Bacterial and fungal community profiling. To profile bacterial and fungal microbiota, 16S rRNA gene and internal transcribed spacer (ITS) DNA library for Illumina sequencing were prepared through two-step PCR amplification protocol. Briefly, DNA concentration for each sample was adjusted to $50 \mathrm{ng} / \mu \mathrm{l}$ (NanoDrop 2000c, Thermo Fisher Scientific). Each sample was amplified two independent times and kept so during the whole procedure indicated in the following. Bacterial DNA was amplified using the forward primer 799F (5'-AACMGGATTAGATACCCKG-3') and reverse primer 1192R (5'-ACGTCATCCCCACCTTCC-3'). To block plant mitochondrial DNA, 10 times the volume of $799 \mathrm{~F}$ or $1193 \mathrm{R}$ of the blocking primer (5'-GGCAAGTGTTCTTCGGA/3SpC3/-3') was added to each reaction. Fungal DNA was amplified using the primers set ITS1F (5'-CTTGGTCATTTAGAGGAAGTAA-3') and ITS1R (5'-GCTGCGTTCTTCATCGATGC-3'). Both 16S rRNA and ITS genes were amplified by PCR in triplicate $\left(95^{\circ} \mathrm{C} / 2\right.$ min; $95^{\circ} \mathrm{C} / 30 \mathrm{~s}, 55^{\circ} \mathrm{C} / 30 \mathrm{~s}, 72^{\circ} \mathrm{C} / 45 \mathrm{~s}$ for 25 cycle; and $72^{\circ} \mathrm{C} / 10$ $\mathrm{min})$. Technical replicates were pooled and leftover primers were enzymatically digested for $30 \mathrm{~min}$ at $37^{\circ} \mathrm{C}$ by adding $3 \mu \mathrm{l}$ of Exonuclease I (20 U), $3 \mu$ l of Antarctic phosphatase (5 U), and $7.32 \mu \mathrm{l}$ of Antarctic phosphatase buffer (both New England BioLabs, Germany). The reaction was heat inactivated at $85^{\circ} \mathrm{C}$ for 15 min. A unique barcode sequence (reverse Illumina compatible primers B1 to B120 and F1 to F120 for bacteria and fungi, respectively) was added to the amplicons obtained from the first PCR reactions over $10 \mathrm{PCR}$ cycles $\left(95^{\circ} \mathrm{C} / 2 \mathrm{~min} ; 95^{\circ} \mathrm{C} / 30 \mathrm{~s}, 55^{\circ} \mathrm{C} / 30 \mathrm{~s}\right.$, $72^{\circ} \mathrm{C} / 45 \mathrm{~s}$; and $\left.72^{\circ} \mathrm{C} / 10 \mathrm{~min}\right)$. All PCR reactions were performed using Q5 high-fidelity DNA polymerase (2,000 units/ml) (New England Biolabs) in triplicate. For 16S rRNA gene amplicons, PCR replicates from second reaction were pooled and run through $1.5 \%$ (wt/vol) agarose gel (90V for ca. $2 \mathrm{~h}$ and $30 \mathrm{~min}$ ) and a band width size of ca. $0.3 \mathrm{~kb}$ was extracted using QIAquick Gel Extraction Kit (Qiagen, Germany) according to the manufacturer's protocol. For ITS amplicons, PCR replicates were pooled and remaining primers were enzymatically digested as indicated above and purified using QIAquick PCR Purification Kit (Qiagen). PCR products were quantified (NanoDrop 2000c) and concentration was adjusted to $20 \mathrm{ng} / \mu \mathrm{l}$ prior to be pooled. Bacterial and fungal PCR amplicon libraries were cleaned twice with Agencourt AMPure XP Kit (Beckman Coulter, Germany) and submitted for DNA sequencing using the MiSeq Reagent kit v3 with the $2 \times 300$ bp paired-end sequencing protocol (Illumina Inc., U.S.A.).

Sequence processing and community analysis. Forward and reverse reads were joined, demultiplexed using Qiime2 pipeline (2018.11.0) (Bolyen et al. 2019). PhiX and chimeric sequences were filtered out using Qiime2-DADA2 (Callahan et al. 2016). Scripts used for sequence processing are available under https:// github.com/hmamine/MWDW/tree/master/reads_preprocessing, and raw MiSeq reads are accessible using the following link: https:// www.ncbi.nlm.nih.gov/bioproject/PRJNA590366. For Shannon and observed operational taxonomic unit (OTU) analyses, count reads were rarefied to an even sequencing depth based on the smallest sample size for bacterial communities and not rarefied for fungal communities using the R package "phyloseq" (McMurdie and Holmes 2013). The R package "picante" (Kembel et al. 2010) 
was used to compute Faith's phylogenetic diversity of rarefied bacterial reads. Prior to computing Bray-Curtis or weighted UniFrac distances, count reads were normalized by cumulative sum scaling normalization factors (Paulson et al. 2013). To test for significantly enriched OTUs, the data were fitted to a zero-inflated Gaussian mixture model (Paulson et al. 2013). To compute neutral and beta-null community assembly models, $\mathrm{R}$ scripts were adapted from Sloan et al. (2006) and Lee et al. (2017), respectively. R scripts used in these analyses are provided via GitHub at https://github. com/hmamine/MWDW/tree/master/community_analysis.

\section{RESULTS}

Diversity of bacteria and fungi across wild and domesticated wheat species. To compare the microbiota between wheat species, T. boeoticum, T. urartu, and T. aestivum were grown in an agricultural soil under controlled growth conditions. After 10 days, bacterial and fungal communities associated with leaves, roots, and rhizosphere of each individual plant, as well as unplanted soil, were profiled by sequencing of the microbial maker genes 16S rRNA and ITS (described in Methods section). The analysis of the community composition indicated that leaf and rhizosphere habitats of $T$. urartu harbor more diverse bacterial communities than T. boeoticum and T. aestivum (Fig. 1A). Furthermore, the communities associated with $T$. urartu showed a trend toward an increased evenness (higher Shannon index) (Fig. 1B) and phylogenetic breadth (Fig. 1C). In contrast to bacteria, the species richness and evenness of fungi was more similar for the three wheat species, and only higher in roots of $T$. urartu, compared with roots of $T$. boeoticum and $T$. aestivum (Fig. 1D and E). Several bacteria belonging to Acidobacteria, Firmicutes, and Proteobacteria mediated the higher alpha-diversity observed in the rhizosphere of T. urartu (Supplementary Fig. S1), whereas the increased fungal diversity in the roots of $T$. urartu was explained by species of diverse groups such as Ascomycota, Basidiomycota, Mortierellomycota, and other fungal phyla (Supplementary Fig. S2). The finding that $T$. boeoticum and T. aestivum harbor comparably diverse microbiota indicates that wheat domestication did not systematically impair microbial diversity and reveals that microbial richness can differ considerably also among wild species.

Plant habitat and genetic identity effects on the structure of the wheat microbiota. To further address how plant habitat (i.e., leaf, root or rhizosphere) and host genetics shape the structure of the microbiota, we projected Bray-Curtis distances between all samples using an ordination method (see Methods). Plant habitat and second wheat genetic identity had significant effect on the assembly of the bacterial and fungal communities (Supplementary Figs. S3 and S4, respectively). Remarkably, the structure of the bacterial communities associated with the rhizosphere of T. urartu was distinct from those of T. boeoticum and T. aestivum (Supplementary Fig. S5A and $\mathrm{B}$ ). However, the corresponding rhizosphere fungal microbiota of these wheat species did not follow similar patterns (Supplementary Fig. 5C), and these results suggest that root exudations of T. urartu to a higher extent favor the enrichment of bacteria. Whether this phenotype is mediated by an increased exudation of "simple" sugars by T. boeoticum as in T. aestivum (Shaposhnikov et al. 2016) remains hypothetical and will require future testing.

To reveal bacterial or fungal taxa that contribute to the observed differences in the community structure, we tested the enrichment of bacterial and fungal OTUs in one wheat species compared with another (see Methods). We show that the observed difference in the community structure of bacteria across the three wheat species is explained by the enrichment of several OTUs (Supplementary Figs. S6, S7, and S8), these OTUs belong mainly to Proteobacteria, Actinobacteria, Firmicutes and Bacteroidetes (Supplementary Fig.
S9). Differences in the structure of fungal communities were less pronounced (Supplementary Fig. S10 to S12). In contrast to bacteria (Supplementary Fig. S9), no single fungal taxa, among the significantly enriched OTUs, was shared between root and rhizosphere habitats (Supplementary Fig. S13). These data indicate that fungi, unlike bacteria, do not follow the multistep microbiota establishment (Bulgarelli et al. 2013; Edwards et al. 2015) and point to a more pronounced role of dispersal in the assembly of the bacterial microbiota compared with the fungi. Taken together, these data indicate that plant habitat and host genetics are selective factors that govern the assembly of the bacterial and fungal microbiota of wild and domesticated wheat species. The interaction of these two factors contributes to the assembly of distinct microbial communities.

Homogeneity of the community structure of the bacterial and fungal microbiota. Wild and domesticated wheat species assemble distinct bacterial and fungal communities. These communities show different degrees of variance in their structure (Özkurt et al. 2019). To compare community structure homogeneity across the microbiota of wild and domesticated wheat species, we computed BrayCurtis (for bacteria and fungi) and weighted UniFrac (only for bacteria) distances to the centroid (see Methods). Bacterial and fungal communities associated with the leaves of $T$. urartu showed less heterogeneity than communities of $T$. boeoticum and $T$. aestivum (Fig. 2A and B). Additionally, leaf-associated bacteria of both $T$. boeoticum and T. urartu showed increased phylogenetic clustering compared with the leaf-associated bacteria of T. aestivum (Fig. 2B). These data indicate that the wild wheat $T$. urartu tends to assemble more homogeneous bacterial- and leaf-associated fungal communities. The current analysis also reveals a substantial relative contribution of stochastic processes in the assembly of the microbiota.

Community assembly processes of the bacterial microbiota of wheat. To assess the relative contribution of stochasticity in the assembly of the microbiota on wild and domesticated wheat, we fitted bacterial data to the Sloan neutral model (Sloan et al. 2006, described in Methods section). By defining soil bacteria as seed source (Venkataraman et al. 2015), we showed that the assembly of the bacterial wheat microbiota do not, or very poorly, follow neutral processes (Supplementary Table S1). This finding additionally corroborates the role of selection in structuring microbial communities. Next, we constrained our analysis to within-group bacterial communities (i.e., across replicates of a same wheat species and corresponding plant habitat, soil bacteria were not defined as seed-source) and quantified ecological drift $\left(R^{2}\right.$ value) and dispersal (computed as immigration coefficient to the model, $m$ value). Except for the phyllosphere of $T$. aestivum, the fit values to the neutral model were overall higher in the unplanted soil and rhizosphere compared with the root and leaf bacterial communities (Fig. 3A, $x$-axis; Supplementary Table $\mathrm{S} 2, R^{2}$ value). Species richness of the communities did not correlate significantly with increased neutrality in the community assembly (Spearman correlation, $\rho=$ $0.503, P$ value $=0.143$ ). Similarly, unplanted soil and rhizosphere habitats exhibited higher immigration coefficients (Fig. 3A, $y$-axis; Supplementary Table $\mathrm{S} 2, m$ value). By comparing the fit and immigration values to the model across plant habitats, we conclude that root- and leaf-associated bacteria are more prone to selection than rhizosphere communities, which suggests that additional plantrelated, and possible unrelated, cues contribute to these processes. The relative contribution of neutral processes in the assembly of the microbiota not only depended on plant habitat, but was also reflected by the wheat genetic identity. Remarkably, neutral processes are better at explaining the assembly of the root and leaf microbiota of the domesticated wheat $T$. aestivum than of the two wild relatives (Fig. $3 \mathrm{~A}, x$-axis; Supplementary Table S2, $R^{2}$ value). The explanation of these findings resides on the fact that fewer bacterial OTUs deviated 
from neutral expectations in T. aestivum (Supplementary Fig. S14, unfilled circles), whereas more OTUs were reported above or below neutral prediction (i.e., over- or under-represented, respectively) in both T. boeoticum (Supplementary Fig. S15) and T. urartu
(Supplementary Fig. S14). Taken together, this analysis indicates that neutral processes have a more prominent role in the assembly of the root and leaf bacterial microbiota of the domesticated wheat T. aestivum compared with its wild relatives.
A

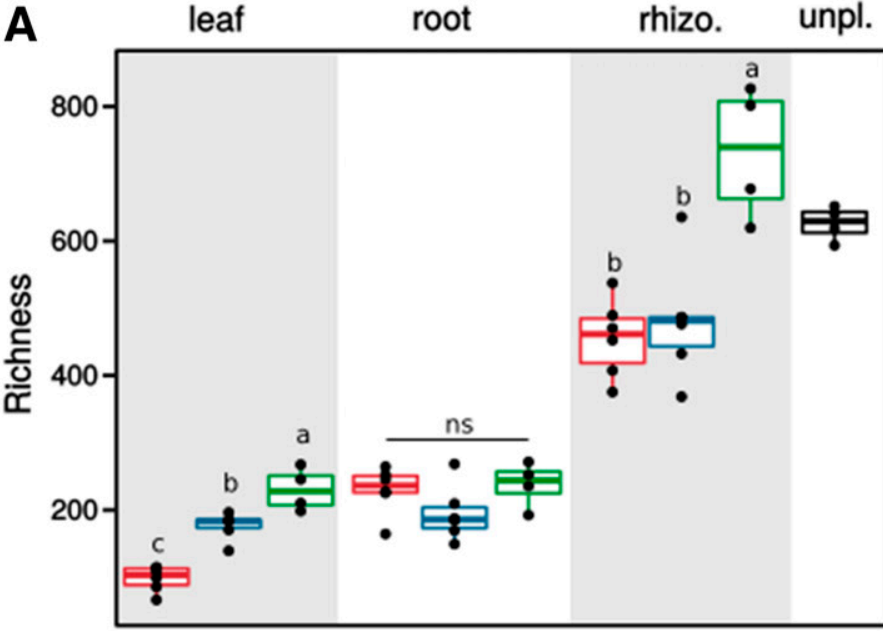

B

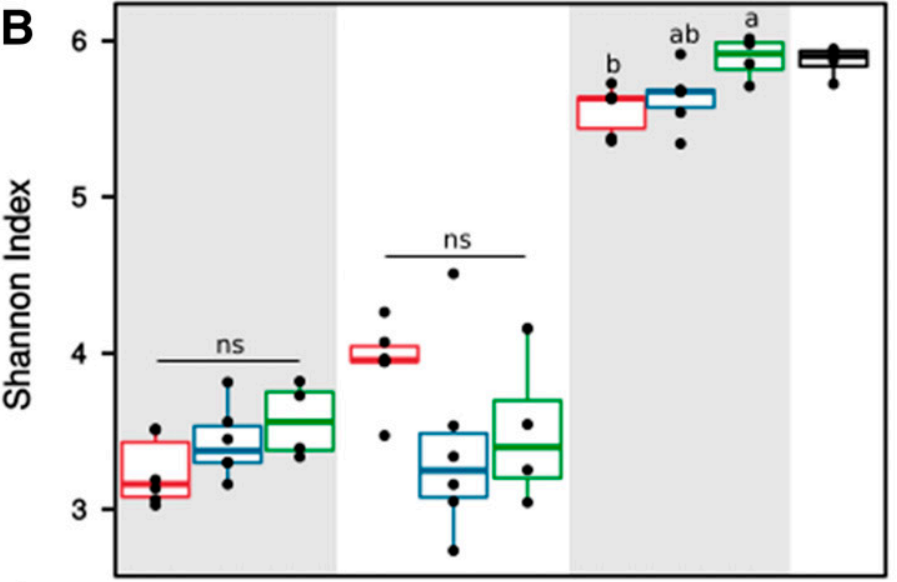

C

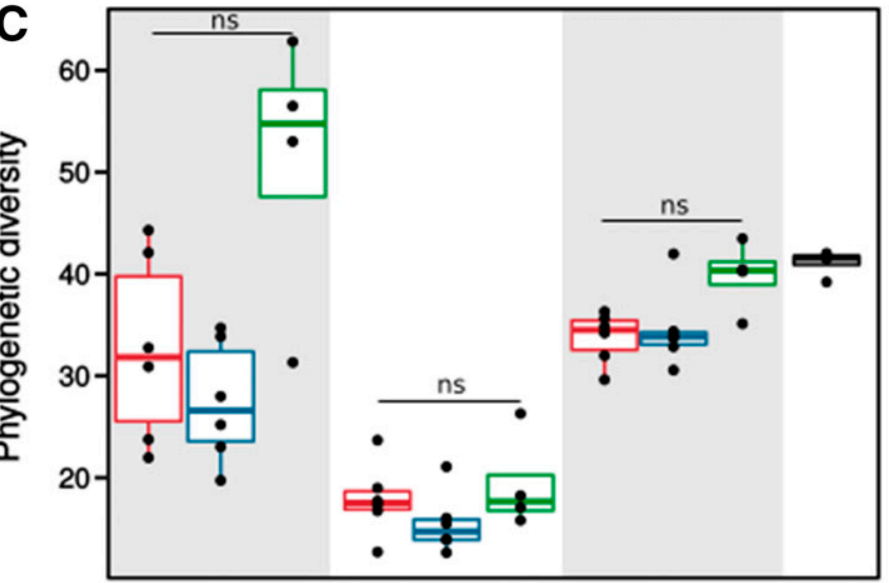

Bacteria
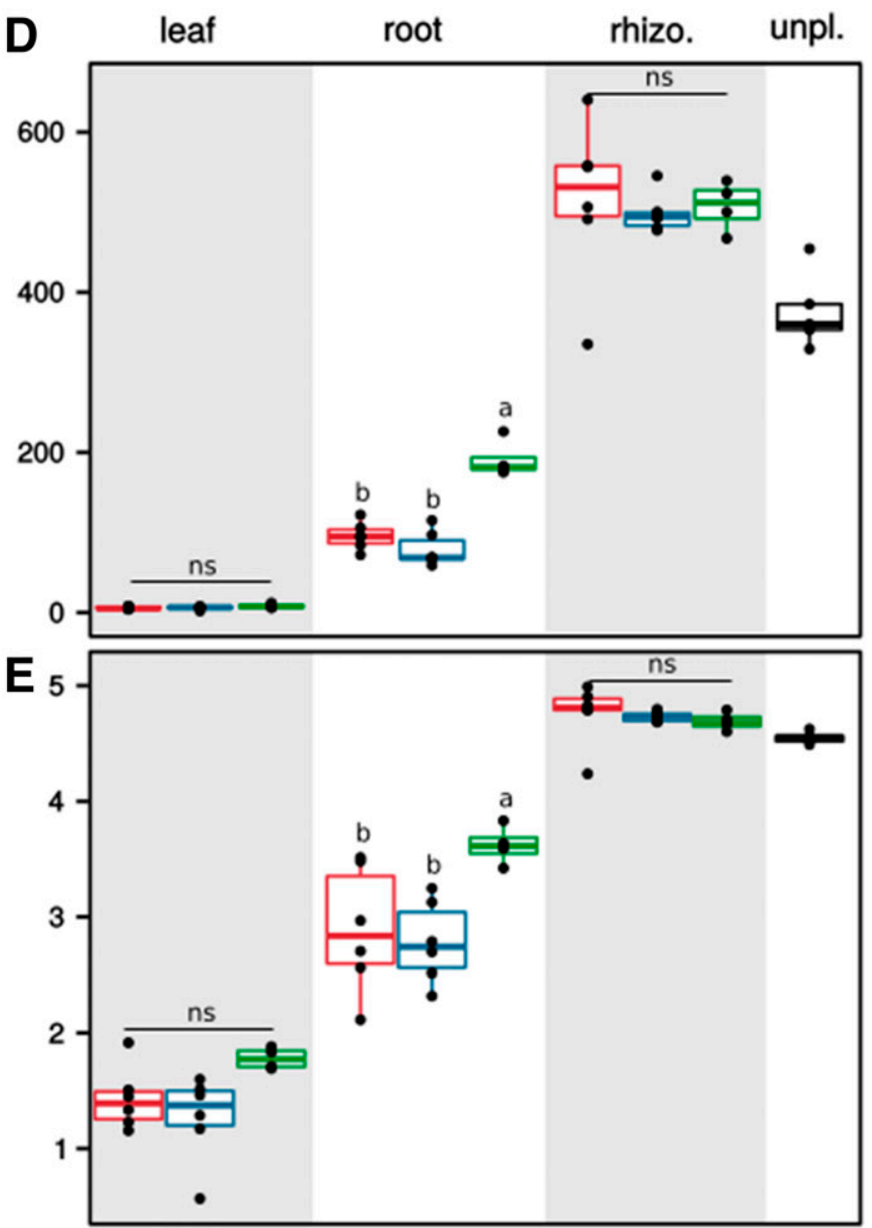

T. aestivum

T. boeoticum

T. urartu

Fig. 1. The diversity of the bacterial and fungal microbiota associated to wild and domesticated wheat species. A, B, C, D, and E, Measures of community diversity of bacterial and fungal microbiota, respectively. A, B, and C, Boxplots that show bacterial community richness and Shannon and Faith's phylogenetic indexes (rarefied to 3,649 reads), respectively. Leaf and rhizosphere habitats of wild wheat Triticum urartu harbor more diverse bacterial communities than $T$. boeoticum and $T$. aestivum, communities which tend to be phylogenetically diverse. Across plant habitats, species evenness is fairly comparable between the three wheat genotypes. D and E, Fungal community richness and Shannon index, respectively. The three wheat genotypes do not show significant differences in their mycobiota diversity, except for the roots of $T$. urartu which show increased species richness and evenness compared with $T$. boeoticum and $T$. aestivum. Color in the graph indicates the wheat genotype, and black boxplot refers to unplanted soil samples. Differences between group medians were tested using Kruskal-Wallis significance test and Conover's multiple comparisons test. rhizo. and unpl. indicate rhizosphere and unplanted soil samples, respectively. 
Role of neutral processes in the assembly of the fungal microbiota of wheat. To quantify the relative contribution of niche and neutral processes in the assembly of the fungal microbiota of wheat, we opted to compute Bray-Curtis beta-diversity deviations from null expectation (Lee et al. 2017; Tucker et al. 2016). Irrespective of the wheat species, fungal communities associated with root and rhizosphere deviated further from null expectations than the leaf fungal microbiota that fell closer to niche processes (Fig. 3B). Across wheat species, neither leaf nor rhizosphere fungal microbiota showed a signification difference in the beta-null deviation. However, the assembly of fungal communities on the roots of $T$. urartu was more prone to niche processes compared with T. boeoticum and T. aestivum. The analysis of Bray-Curtis deviation from null model indicates that the transition from the rhizosphere through the root to the leaf habitat is concomitant with a decrease in niche processes. Remarkably, similar analysis conducted on the bacterial communities showed the opposite, i.e., an increase in niche processes (Fig. 3C). We conclude from these results that the assembly of bacterial and fungal communities follows distinct community patterns and further suggest that wheat domestication and breeding have to a lesser extent altered the assembly of the wheat fungal microbiota. The divergent patterns are most likely mediated by the constrained dispersal of fungi (Supplementary Fig. S15) and the scarcity of dominant fungal OTUs across individual plants (Supplementary Fig. S16).

\section{DISCUSSION}

In this study, we have investigated bacterial and fungal communities that assemble on wild and domesticated wheat species.
A

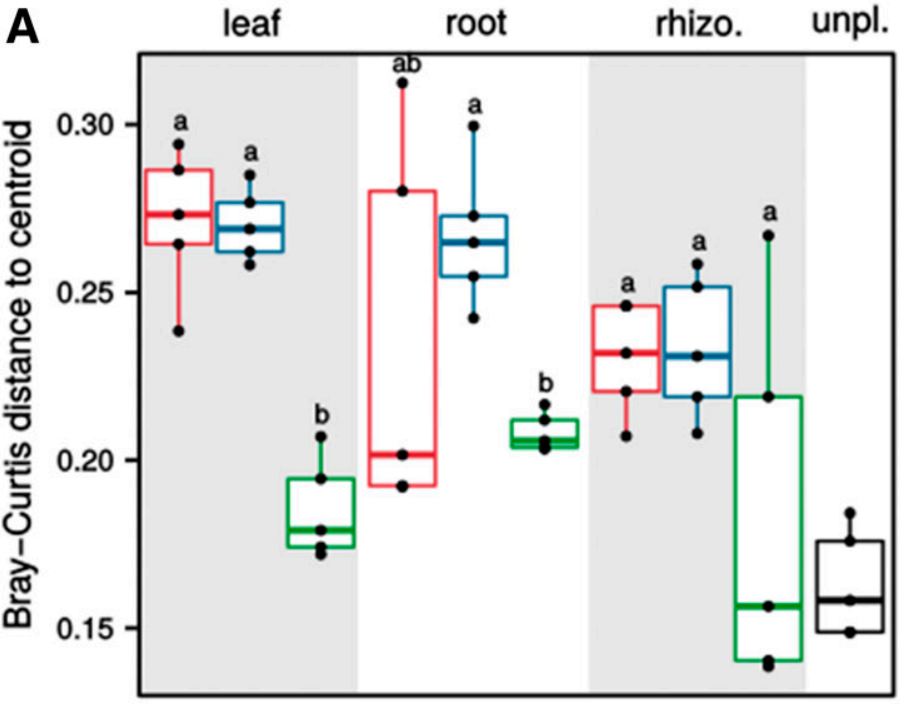

B

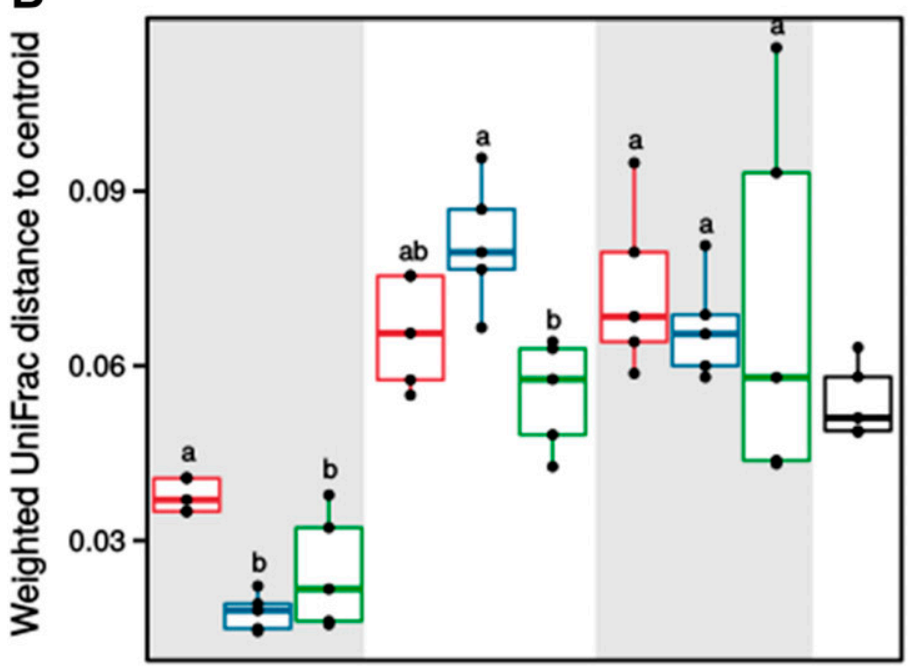

Bacteria

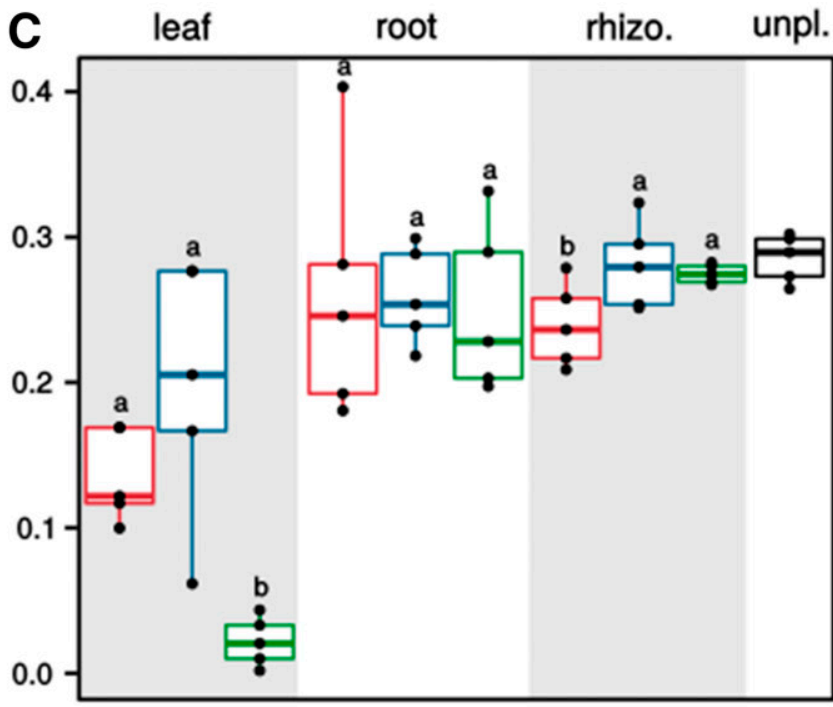

T. aestivum
T. boeoticum

T. urartu

Fig. 2. Heterogeneity in structure of bacterial and fungal wheat microbiota. A and B, Community homogeneity analyses depicted by Bray-Curtis and weighted UniFrac distances to centroid for the bacterial microbiota, respectively. A, The decrease in Bray-Curtis distance to centroid indicates that the leaf and root microbiota of the wild wheat Triticum urartu assemble more homogeneous bacterial communities than T. boeoticum and T. aestivum. B, Both leaf communities associated to $T$. boeoticum and $T$. urartu are more phylogenetically clustered than bacterial communities associated to $T$. aestivum. C, Across root and rhizosphere habitat, wheat genotype do not explain fungal community heterogeneity. Leaf fungal microbiota of $T$. urartu shows greater increased homogeneity than the fungal microbiota associated to $T$. boeoticum and T. aestivum. Count data were normalized using cumulative sum scaling factor and operational taxonomic units, with occurrences $<3$ trimmed out prior to computing distances. Differences in group means were tested using Tukey's honest test ( 0.95 confidence level). rhizo. and unpl. indicate rhizosphere and unplanted soil samples, respectively. 
Microbial richness of wild and domesticated wheat species was assessed across habitats and microbial kingdoms, we reported a tendency of the wild wheat $T$. urartu to harbor more diverse microbiota. Bacterial communities associated with the leaves and rhizosphere were more diverse in $T$. urartu than in $T$. boeoticum and T. aestivum. Although both T. urartu and T. boeoticum are wild relatives of wheat, we showed a discrepancy in microbial richness between the two aforementioned wild wheat species.

Did plant domestication lead to a loss of microbial diversity? Our findings join other studies that have revealed less diverse microbial communities associated with modern crop species (Coleman-Derr et al. 2016; Zachow et al. 2014). For example, Coleman-Derr et al.
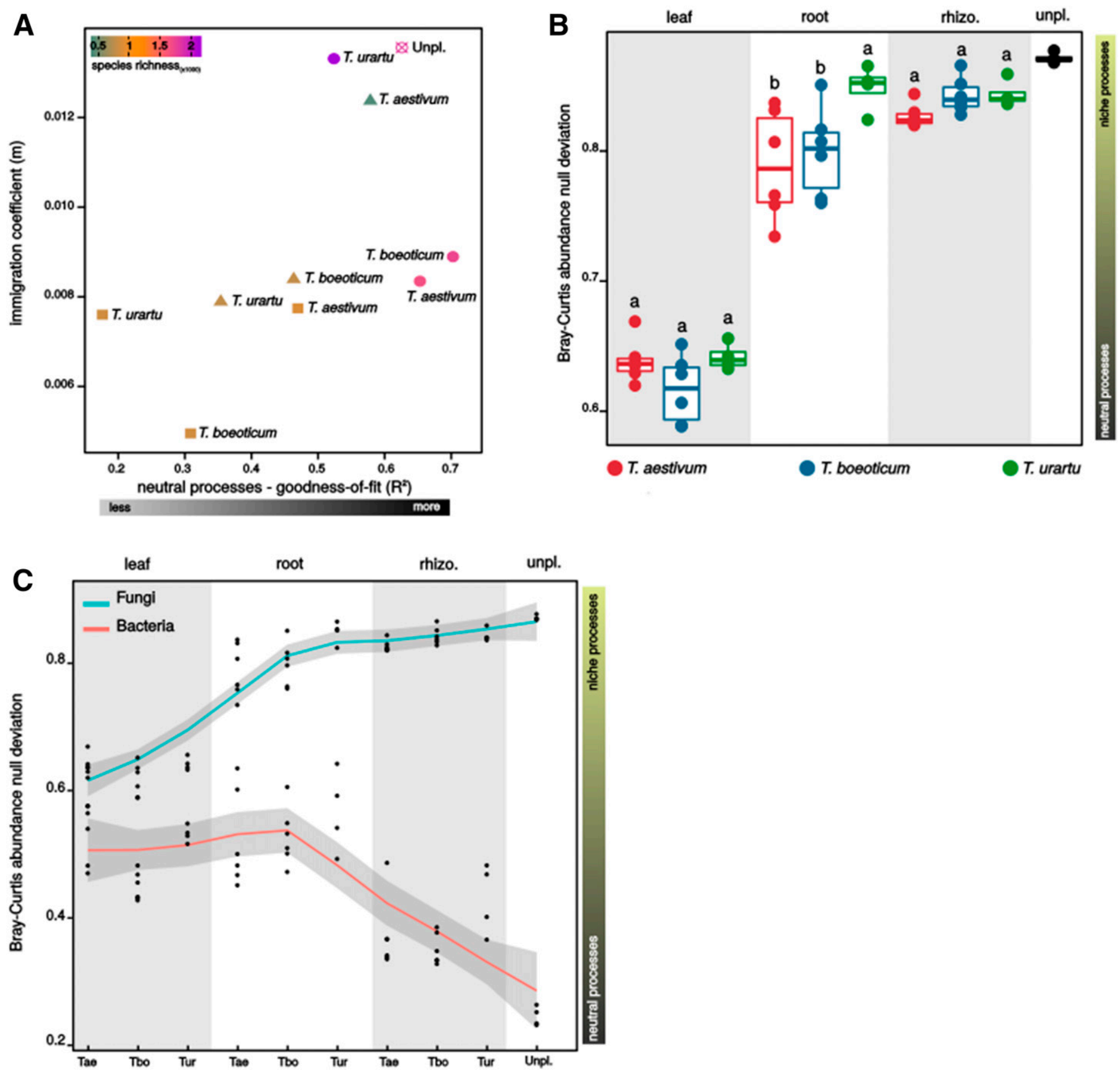

Fig. 3. Ecological assembly processes of the bacterial and fungal microbiota of wheat. A, Scatter-plot depicts the goodness of fit $\left(R^{2}\right)$ and immigration coefficient $(\mathrm{m})$ to the neutral model of bacterial communities. In the graph, square, triangle, and circle indicate root, leaf, and rhizosphere bacterial communities, respectively. Unplanted soil is indicated by a crossed unfilled circle. Color gradient in the shapes indicates species richness. The root and leaf microbiota of wild wheat show decreased fit to the neutral processes. The root and leaf bacterial microbiota of wild wheat show a lower fit to the neutral model than Triticum aestivum. B, Boxplot depicts Bray-Curtis deviation from the null model of the fungal microbiota of wheat across leaf, root, and rhizosphere (rhizo.) habitats and unplanted (unpl.). The root fungal microbiota of $T$. boeoticum and $T$. aestivum show further deviation from niche processes than the fungal microbiota of $T$. urartu. Significance within-group medians were tested using Kruskal-Wallis significance and Conover's multiple comparisons tests. C. The plot depicts Bray-Curtis deviation from the null model of the bacterial and fungal microbiota of wheat across leaf, root, and rhizosphere habitats. Color in the line indicates the microbial kingdom. Lines show local regression fitting (Loess, $x \sim y, 0.95$ confidence interval) in the Bray-Curtis null deviations. Singletons were trimmed out from the count data prior to the model computation. 
(2016) showed that native Agave species harbor more diverse bacterial and fungal communities in the rhizosphere and phyllosphere than the cultivated species Agave tequilana (Coleman-Derr et al. 2016). Conversely, Leff et al. (2017) showed that the diversity of bacterial communities associated with the root or rhizosphere of wild, native American or modern sunflower (Helianthus annuus) did not show significant differences, but the extend of sunflower domestication did affect the composition of the rhizosphere fungal microbiota (Leff et al. 2017). In the current study, we could show that only the root fungal diversity was significantly higher in $T$. urartu compared with $T$. boeoticum or $T$. aestivum. Conflicting empirical evidences about the effect of crop domestication on the diversity of the plant microbiota indicate that a potential "domestication syndrome" (Cordovez et al. 2019; Doebley et al. 2006) is mitigated and depends on the crop species. Furthermore, the soil type is a factor that also may lead to different observations. In the recent study of Pérez-Jaramillo et al. (2019), the authors showed that wild landrace and modern bean accessions (Phaseolus vulgaris) differed significantly in the compositions of the rhizosphere bacterial communities only when grown in agricultural, but not native soil (PérezJaramillo et al. 2019). Although the effect of crop domestication on the microbiota diversity is not clear, these studies, including the present one, have shown that host genetic identity plays an important role in shaping the microbiota structure (i.e., microbial species composition and their relative abundance) and indicate that host cues are selective factors in the assembly of the plant microbiota.

By deciphering ecological processes in the assembly of the microbiota, we showed that neutral processes have a more prominent role in the assembly of the bacterial microbiota of T. aestivum. Although T. urartu tends to harbor a more diverse bacterial microbiota while T. boeoticum and T. aestivum showed comparably diverse bacterial communities of roots and rhizospheres, the goodness-of-fit to the Sloan neutral model of bacterial communities of roots and leaves were lower in T. urartu and $T$. boeoticum compared with $T$. aestivum. This finding suggests that wild wheat exert more selection on the assembly of its microbiota. In contrast, we report little to no effect of wheat genotype on the assembly of the fungal microbiota. Notably, the structure of the fungal microbiota did significantly differ between the three wheat species. A possible explanation is that domestication has altered mechanisms involved in assembly of the bacterial, but not fungal, communities. These mechanisms could be mediated by different selective factors including plant physiology, immune-related traits, nutrient concentrations, and chemical composition of root exudation. Alternatively, this pattern could be a result of constrained dispersal of fungi. These two hypotheses are not mutually exclusive. It is likely that the plant controls the assembly the fungal microbiota as protective mechanisms against fungal pathogens (Leff et al. 2017; Tkacz et al. 2020).

In conclusion, based on our findings, we propose that domestication might have relaxed selective processes that govern the assembly of the bacterial microbiota in the root and leaf habitats of T. aestivum. Whether this phenotype is related to a loss of genetic cues in modern wheat will require future testing.

\section{ACKNOWLEDGMENTS}

We thank Janine Haueisen and Michael Habig for helpful comments to an earlier version of this manuscript.

\section{LITERATURE CITED}

Bolyen, E., Rideout, J. R., Dillon, M. R., Bokulich, N. A., Abnet, C. C., AlGhalith, G. A., et al. 2019. Reproducible, interactive, scalable and extensible microbiome data science using QIIME 2. Nat. Biotechnol. 37:852-857.
Bulgarelli, D., Schlaeppi, K., Spaepen, S., van Themaat, E. V. L., and SchulzeLefert, P. 2013. Structure and functions of the bacterial microbiota of plants. Annu. Rev. Plant Biol. 64:807-838.

Callahan, B. J., McMurdie, P. J., Rosen, M. J., Han, A. W., Johnson, A. J. A., and Holmes, S. P. 2016. DADA2: High resolution sample inference from Illumina amplicon data. Nat. Methods 13:581-583.

Coleman-Derr, D., Desgarennes, D., Fonseca-Garcia, C., Gross, S., Clingenpeel, S., Woyke, T., North, G., Visel, A., Partida-Martinez, L. P., and Tringe, S. G. 2016. Plant compartment and biogeography affect microbiome composition in cultivated and native Agave species. New Phytol. 209:798-811.

Cordovez, V., Dini-Andreote, F., Carrión, V. J., and Raaijmakers, J. M. 2019. Ecology and evolution of plant microbiomes. Annu. Rev. Microbiol. 73: 69-88.

Diamond, J. 2002. Evolution, consequences and future of plant and animal domestication. Nature 418:700-707.

Doebley, J. F., Gaut, B. S., and Smith, B. D. 2006. The molecular genetics of crop domestication. Cell 127:1309-1321.

Edwards, J., Johnson, C., Santos-Medellín, C., Lurie, E., Podishetty, N. K., Bhatnagar, S., Eisen, J. A., and Sundaresan, V. 2015. Structure, variation, and assembly of the root-associated microbiomes of rice. Proc. Natl. Acad. Sci. USA 112:E911-E920.

Escudero-Martinez, C., and Bulgarelli, D. 2019. Tracing the evolutionary routes of plant-microbiota interactions. Curr. Opin. Microbiol. 49:34-40.

Fuller, D. Q. 2007. Contrasting patterns in crop domestication and domestication rates: Recent archaeobotanical insights from the Old World. Ann. Bot. 100: 903-924.

Hassani, M. A., Özkurt, E., Seybold, H., Dagan, T., and Stukenbrock, E. H. 2019. Interactions and coadaptation in plant metaorganisms. Annu. Rev. Phytopathol. 57:483-503.

Kembel, S. W., Cowan, P. D., Helmus, M. R., Cornwell, W. K., Morlon, H., Ackerly, D. D., Blomberg, S. P., and Webb, C. O. 2010. Picante: R tools for integrating phylogenies and ecology. Bioinformatics 26:1463-1464.

Kiers, E. T., Hutton, M. G., and Denison, R. F. 2007. Human selection and the relaxation of legume defences against ineffective rhizobia. Proc. Roy. Soc. B: Biol. Sci. 274:3119-3126.

Lee, S.-H., Sorensen, J. W., Grady, K. L., Tobin, T. C., and Shade, A. 2017. Divergent extremes but convergent recovery of bacterial and archaeal soil communities to an ongoing subterranean coal mine fire. ISME J. 11: 1447-1459.

Leff, J. W., Lynch, R. C., Kane, N. C., and Fierer, N. 2017. Plant domestication and the assembly of bacterial and fungal communities associated with strains of the common sunflower, Helianthus annuus. New Phytol. 214:412-423.

McMurdie, P. J., and Holmes, S. 2013. phyloseq: An R package for reproducible interactive analysis and graphics of microbiome census data. PLoS One 8: e61217.

Nemergut, D. R., Schmidt, S. K., Fukami, T., O’Neill, S. P., Bilinski, T. M., Stanish, L. F., Knelman, J. E., Darcy, J. L., Lynch, R. C., Wickey, P., and Ferrenberg, S. 2013. Patterns and processes of microbial community assembly. Microbiol. Mol. Biol. Rev. 77:342-356.

Özkurt, E., Hassani, M. A., Sesiz, U., Künzel, S., Dagan, T., Özkan, H., and Stukenbrock, E. H. 2019. Higher stochasticity of microbiota composition in seedlings of domesticated wheat compared to wild wheat. bioRxiv 685164 .

Herrera-Paredes, S. H., and Lebeis, S. L. 2016. Giving back to the community: Microbial mechanisms of plant-soil interactions. Funct. Ecol. 30:1043-1052.

Paulson, J. N., Stine, O. C., Bravo, H. C., and Pop, M. 2013. Differential abundance analysis for microbial marker-gene surveys. Nat. Methods 10: 1200-1202.

Pérez-Jaramillo, J. E., Carrión, V. J., de Hollander, M., and Raaijmakers, J. M. 2018. The wild side of plant microbiomes. Microbiome 6:143.

Pérez-Jaramillo, J. E., de Hollander, M., Ramírez, C. A., Mendes, R., Raaijmakers, J. M., and Carrión, V. J. 2019. Deciphering rhizosphere microbiome assembly of wild and modern common bean (Phaseolus vulgaris) in native and agricultural soils from Colombia. Microbiome 7:114.

Pont, C., Leroy, T., Seidel, M., Tondelli, A., Duchemin, W., Armisen, D., et al. 2019. Tracing the ancestry of modern bread wheats. Nat. Genet. 51:905-911.

Preece, C., Livarda, A., Christin, P.-A., Wallace, M., Martin, G., Charles, M., Jones, G., Rees, M., and Osborne, C. P. 2017. How did the domestication of Fertile Crescent grain crops increase their yields? Funct. Ecol. 31:387-397.

Purugganan, M. D., and Fuller, D. Q. 2009. The nature of selection during plant domestication. Nature 457:843-848.

Shaposhnikov, A. I., Morgounov, A. I., Akin, B., Makarova, N. M., Belimov, A. A., and Tikhonovich, I. A. 2016. Comparative characteristics of root systems and root exudation of synthetic, landrace and modern wheat varieties. Agric. Biol. 68-78. 
Sloan, W. T., Lunn, M., Woodcock, S., Head, I. M., Nee, S., and Curtis, T. P. 2006. Quantifying the roles of immigration and chance in shaping prokaryote community structure. Environ. Microbiol. 8:732-740.

Tkacz, A., Pini, F., Turner, T. R., Bestion, E., Simmonds, J., Howell, P., Greenland, A., Cheema, J., Emms, D. M., Uauy, C., and Poole, P. S. 2020. Agricultural selection of wheat has been shaped by plant-microbe interactions. Front. Microbiol. 11:132.

Tucker, C. M., Shoemaker, L. G., Davies, K. F., Nemergut, D. R., and Melbourne, B. A. 2016. Differentiating between niche and neutral assembly in metacommunities using null models of $\beta$-diversity. Oikos 125 : 778-789.
Vellend, M., Srivastava, D. S., Anderson, K. M., Brown, C. D., Jankowski, J. E., Kleynhans, E. J., Kraft, N. J. B., Letaw, A. D., Macdonald, A. A. M., Maclean, J. E., Myers-Smith, I. H., Norris, A. R., and Xue, X. 2014. Assessing the relative importance of neutral stochasticity in ecological communities. Oikos 123:1420-1430.

Venkataraman, A., Bassis, C. M., Beck, J. M., Young, V. B., Curtis, J. L., Huffnagle, G. B., and Schmidt, T. M. 2015. Application of a neutral community model to assess structuring of the human lung microbiome. MBio 6:e02284-e14.

Zachow, C., Müller, H., Tilcher, R., and Berg, G. 2014. Differences between the rhizosphere microbiome of Beta vulgaris ssp. maritima - ancestor of all beet crops-and modern sugar beets. Front. Microbiol. 5:415. 